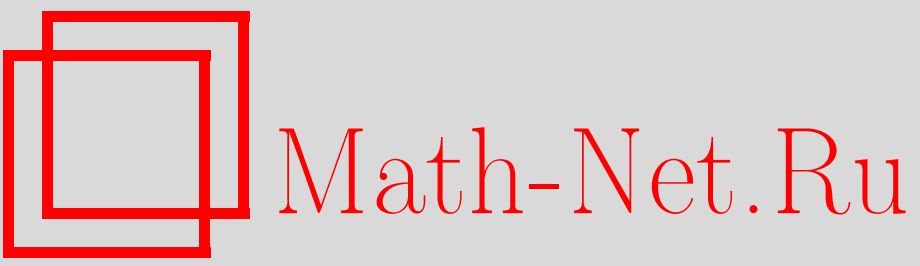

А. В. Головашкин, В. М. Максимов, О косых полиномах Оре высших порядков, порождаемых однородными квадратичными соотношениями, УМH, 1998, том 53, выпуск 2, 145-146

DOI: https://doi.org/10.4213/rm36

Использование Общероссийского математического портала Math-Net.Ru подразумевает, что вы прочитали и согласны с пользовательским соглашением

http: //www . mathnet.ru/rus/agreement

Параметры загрузки:

IP: 34.239 .49 .27

26 апреля 2023 г., 17:59:41 


\title{
О КОСЫХ ПОЛИНОМАХ ОРЕ ВЫСШИХ ПОРЯДКОВ, ПОРОЖДАЕМЫХ ОДНОРОДНЫМИ КВАДРАТИЧНЫМИ СООТНОШЕНИЯМИ
}

\author{
А. В. ГоловАшкИн, В. М. МАКСИмов
}

1. Алгебра косых полиномов Оре хорошо известна. Она состоит из однозначно представляемых элементов $\alpha_{0}+\alpha_{1} \tau+\cdots+\alpha_{k} \tau^{k}, k=k(\alpha)=0,1,2, \ldots, \alpha_{i} \in \mathscr{K}$, где $\mathscr{K}$ - колшцо, с условием перестановки $\tau \alpha=\varphi(\alpha) \tau+\psi(\alpha)$. При этом $\varphi$ является эндоморфизмом колшца $\mathscr{K}$, а $\psi$-его $\varphi$-дифференцирование [1]. В [2]-[5] рассматривались различные обобщения, назьваемые косыми полиномами Оре высших порядков, которые возникают при условии перестановки

$$
\tau \alpha=\psi_{1}(\alpha) \tau+\psi_{2}(\alpha) \tau^{2}+\cdots,
$$

что является удобным способом задания алгебры, так как тогда она определяется набором операторов $\psi_{k}: \mathscr{K} \rightarrow \mathscr{K}$, связанных специальными алгебраическими соотношениями [5].

Однако, имеются случаи, в которых условия перестановки (1) оказываются недостаточными для задания алгебры косыми полиномами. Такими, например, будут алгебры, заданные общим квадратичным соотношением, которые находят важные приложения в физике [6].

При этом оказывается, что алгебры, заданные квадратичным соотношением, представляются косыми полиномами, но с соотношением перестановки в которых добавлен свободный, ненулевой член $\psi_{0}$.

В [7] рассмотрены косые полиномы Оре с условием перестановки со свободньм членом $\psi_{0}$, т.е.

$$
\tau \alpha=\psi_{0}(\alpha)+\psi_{1}(\alpha) \tau+\psi_{2}(\alpha) \tau^{2}+\cdots,
$$

где $\psi_{0}$ при произвольных $\alpha, \beta \in \mathscr{K}$ удовлетворяет соотношению

$$
\psi_{0}(\alpha \beta)=\psi_{0}(\alpha) \beta+\psi_{1}(\alpha) \psi_{0}(\beta)+\psi_{2}(\alpha) \psi_{0}^{2}(\beta)+\cdots
$$

или эквивалентному ему операторному уравнению $\psi_{0} \alpha=\psi_{0}(\alpha)+\psi_{1}(\alpha) \psi_{0}+\psi_{2}(\alpha) \psi_{0}^{2}+\cdots$, в котором $\alpha$ рассматривается как оператор левого умножения на $\alpha$ колца $\mathscr{K}$. Можно было бы считать $\psi_{0}$ особьм оператором диффференцирования относительно $\psi_{1}, \psi_{2}, \ldots$, но при этом $\psi_{1}$ может и не быть эндоморфизмом кольца $\mathscr{K}$.

Цель нашей заметки изложить полученные результаты о представлении алгебр, заданных квадратичным соотношением от двух образующих $a, b$ :

$$
b a=\alpha a^{2}+\beta a b+\gamma b^{2}
$$

в виде алгебры косых полиномов Оре высших порядков с условием перестановки вида (2). При этом основные резултаты остаются справедливыми, если $\alpha, \beta, \gamma$ рассматривать из произвольного поля $F$ характеристики 0 .

2. Алгебра, порождаемая соотношением (3), очевидно, представляется в виде алгебры косых полиномов Оре высших порядков, если элементы $\left\{a^{m} b^{n}\right\}$ образуют линейный базис алгебры. Тогда алгебра, порождаемая (3), может быть определена системой линейных отображений $\psi_{0}, \psi_{1}, \psi_{2}, \ldots$ алгебры полиномов $F(a)$ в себя. Тогда для $p(a) \in F(a)$ имеем: $b p(a)=$ $\psi_{0}(p(a))+\psi_{1}(p(a)) b+\cdots+\psi_{k}(p(a)) b^{k}, k=k(p(a)), k=0,1,2, \ldots$ В предположении, что такое представление действительно существует, легко вьвести соотношения между операторами $\psi_{0}, \psi_{1}, \psi_{2}, \ldots$. Обозначим через $X$ оператор умножения на образующую $a$ в кольце полиномов $F(a)$.

ПРЕДЛОЖЕНИЕ 1. Имеют место равенства

$$
\begin{aligned}
\psi_{0} X & =\alpha X^{2}+\beta X \psi_{0}+\gamma \psi_{0}^{2}, \\
\psi_{k} X & =\beta X \psi_{k}+\gamma\left[\psi_{0} \psi_{k}+\psi_{1} \psi_{k-1}+\cdots+\psi_{k} \psi_{0}\right], \quad k=1,2, \ldots
\end{aligned}
$$

Если вьполняется (4), то уравнение (3) дает $\psi_{0}(a)=\alpha a^{2}$. Тогда из первого уравнения, если оно имеет решение, должно быть $\psi_{0}\left(a^{n}\right)=\bar{\psi}_{0}^{n} a^{n+1}, \bar{\psi}_{0}^{n} \in F$, а всю систему можно дополнить непротиворечивыми начальными условиями $\psi_{0}(\mathbf{1})=0, \psi_{1}(\mathbf{1})=\mathbf{1}, \psi_{k}(\mathbf{1})=0, k \geqslant 2$. Тогда

Работа выполнена при поддержке Российского фонда фундаментальных исследований (грант № 96-01-00492). 
из (4) легко следует, что $\psi_{k}\left(a^{n}\right)=\bar{\psi}_{k}^{n} a^{n-k+1}, n+1 \geqslant k ; \psi_{k}\left(a^{n}\right)=0, k>n+1$, где $\bar{\psi}_{k}^{n} \in F$ константы, определяющие оператор $\psi_{k}$.

Оказьвается, в случае существования решения $\psi_{0}$, все остальные решения $\psi_{k}$ вьражаются через $\psi_{0}$ по формулам, похожим на общие формулы, данные в [3], [4]. Однако, они имеют другой смысл, так как там $\psi_{0}=0$.

Для выражения $\psi_{k}$ нам понадобятся два оператора. Это оператор $V_{0}$ - оператор значения в нуле, известный как $\delta$-функция Дирака, т.е. $V_{0} p(a)=p(0)$, и оператор $\bar{D}$, понижающий степень на единицу, т.е. $\bar{D} \mathbf{1}=0, \bar{D} a^{n}=a^{n-1}, n \geqslant 1$. Для произвольного полинома $p(a)$, очевидно, имеем, $\bar{D} p(a)=\frac{p(a)-p(0)}{a}$. Следовательно, оператор $\bar{D}$ есть $V_{0}$-дифференцирование на $F(a)$.

Относительно коэффициентов $\alpha$ и $\gamma$ мы будем предполагать, что $\alpha \cdot \gamma \neq 0$, так как в случае $\alpha=0$ или $\gamma=0$ уравнение (3) приводит к классическим полиномам Оре над кольцом $F(a)$.

ПрЕДЛОЖЕнИЕ 2. Если уравнение $\psi_{0} X=\alpha X^{2}+\beta X \psi_{0}+\gamma \psi_{0}^{2}$ имеет решение $\psi_{0}$, где $\psi_{0} \mathbf{1}=0, \psi_{0} a^{n}=\bar{\psi}_{0}^{n} a^{n+1}$ при некоторых $\bar{\psi}_{0}^{n} \in F$, то существует единственная система операторов $\psi_{k}$, удовлетворяющая системе (4), и для них имеет место

$$
\psi_{1}=\frac{\beta}{\alpha}\left(\bar{D} \psi_{0}-\psi_{0} \bar{D}\right)+V_{0}=\frac{\beta}{\alpha}\left[\bar{D}, \psi_{0}\right]+V_{0} ; \quad \psi_{k}=\psi_{1} \mathscr{F}^{k-1}, \quad k \geqslant 1,
$$

əде $\mathscr{F}=\frac{\gamma}{\alpha} \bar{D}^{2} \psi_{0}$.

3. В силу этого предложения центральным моментом в решении уравнений (4) является нахождение оператора $\psi_{0}$. Введем для любого столбца $\left(\begin{array}{c}c_{1} \\ c_{2}\end{array}\right), c_{2} \neq 0$, обозначение $\left(\begin{array}{c}c_{1} \\ c_{2}\end{array}\right) \div=c_{1} / c_{2}$. Тогда имеет место

ПРЕДЛОЖЕНИЕ 3. Если правый нижний әлемент матрищы $\left(\begin{array}{cc}\beta & \alpha \\ -\gamma & 1\end{array}\right)^{n}$ для любого $n=$ $1,2, \ldots$ отличен от нуля, то уравнение (4) имеет единственное решение $\psi_{0}$ и для всех $n=1,2, \ldots$

$$
\bar{\psi}_{0}^{n}=\left[\left(\begin{array}{cc}
\beta & \alpha \\
-\gamma & 1
\end{array}\right)^{n} \cdot\left(\begin{array}{l}
0 \\
1
\end{array}\right)\right] \div
$$

Это предложение позволяет сформулировать условия, когда одночлены $\left\{a^{m} b^{n}\right\}, m, n=0$, $1,2, \ldots$, образуют базис алгебры, порождаемой (3). Обозначим определитель матрицы $\left(\begin{array}{cc}\beta & \alpha \\ -\gamma & 1\end{array}\right)$ через $\Delta$.

ПрЕДЛОЖЕНИЕ 4. Если $\Delta \neq 0$, то для того чтобы в алгебре, заданной (3), одночлень $\left\{a^{m} b^{n}\right\}$ образовывали базис, необходимо и достаточно, чтобь нижние правье әлементы матрич $\left(\begin{array}{cc}\beta & \alpha \\ -\gamma & 1\end{array}\right)^{n}, n=1,2,3, \ldots$, были отличны от нуля.

ПреДлОЖенИЕ 5. Если $\Delta=0$, то базис алгебры, порождаемой (3) всегда состоит из одночленов $\left\{a^{m} b^{n}\right\}$, кроме случая $b a=\alpha a^{2}-a b+\frac{1}{\alpha} b^{2}$, когда эти одночлень линейно независимы, но базиса не образуют.

В ряде частных случаев, когда $a^{2}+b^{2}=0 ; \alpha=\beta=0, \gamma=1$ и $\beta=\gamma=0, \alpha=1$, с помощью бриллиантовой леммы в [8] найдены базисы другого вида. Например, в случае $\beta=\gamma=0, \alpha=1$ базис состоит из одночленов $\left\{a^{r}\right\},\left\{a^{m} b a^{n}\right\}$. Однако, из них с помощью соотношения (3) легко получить базис $\left\{a^{m} b^{n}\right\}$.

\section{СПИСОК ЛИТЕРАТУРЫ}

[1] Ore O. // Ann. Math. 1931. V. 32. P. 463-477. [2] Cohn P. M. // Proc. London Math. Soc. 1961. V. 11. № 3. P. 531-556. [3] Smits T. H. M. // Indag. Math. 1968. V. 30. P. 209-224. [4] Кон П. Свободные колца и их связи. М.: Мир, 1975. [5] Dumas F. // Lecture Notes Math. 1991. V. 1478. Р. 192-214. [6] Островский В. Л., Самойленко Ю. С. // Уч. записки ЛОМИ. 1989. Т. 172. С. 121-129. [7] Максимов В. М. Косые полиномы Оре высших порядков. (готовится к печати). [8] Уфонаровский В. А. // Итоги науки и техники. 1990. Т. 57. С. 5-177. 